\title{
Extension of Sub Compatible Maps in Fuzzy Metric Spaces
}

\author{
S.K. Malhotra, Vineeta Singh \\ (Deputy Controller, M.P. Professional Examination Board, Bhopal) \\ ( Asstt. Prof. Dept. of app. maths and computer science,S.A.T.I.,Vidisha)
}

\begin{abstract}
The present paper is the extension of results of sub compatibility and sub sequential continuity in fuzzy metric spaces which are weaker than occasionally weak compatibility and reciprocal continuity.

Mathematics Subject Classification (2000). 47H10, 54H25.

Keywords: Compatible maps, Weak Compatible maps, Occasionally weak compatible maps, Sub compatible maps, , fixed points and fuzzy metric space space
\end{abstract}

\section{Introduction}

The concept of fuzzy set given by Zadeh [15] it was a turning point in the development of Mathematics and laid the foundation of fuzzy mathematics. Consequently, the last three decades remained productive for various authors like Deng [7], Erceg [8], Kaleva and Siekkala [11], Kramosil and Michalek [10], Pant [13], Sessa [14] etc. Various authors have discussed and studied extensively various results on coincidence, existence and uniqueness of fixed and common fixed points by using the concept of weak commutativity, compatibility, non-compatibility and weak compatibility for single and set valued maps satisfying certain contractive conditions in different spaces and they have been applied to diverse problems. Recently, Al-Thagafi and Shahzad [2] weakened the concept of compatibility by giving a new notion of occasionally weakly compatible (owc) maps which is more general among the commutativity concepts. Most recently, Bouhadjera and Thobie [4], weakened the concept of occasionally weak compatibility and reciprocal continuity in the form of sub compatibility and sub sequential continuity respectively and proved some interesting results with these concepts in metric spaces.

\section{Preliminaries}

Definition 2.1. A binary operation $*:[0,1] \times[0,1] \rightarrow[0,1]$ is continuous $t-$ norm if $*$ is satisfying the following conditions:

(i) * is commutative and associative

(ii) $*$ is continuous

(iii) $a * 1=$ a for all $a \in[0,1]$

(iv) $a * b \leq c * d$ whenever $a \leq c$ and $b \leq d, a, b, c, d \in[0,1]$.

Definition 2.2. A triplet $(X, M, *)$ is said to be a fuzzy metric space if $\mathrm{X}$ is an arbitrary set,

* is a continuous $t-$ norm and $\mathrm{M}$ is a fuzzy set on $X^{2} \times(0, \infty)$ satisfying the following;

(FM-1) $M(x, y, t)>0$

(FM-2) $M(x, y, t)=1$ if and only if $x=y$.

(FM-3) $M(x, y, t)=M(y, x, t)$

(FM-4) $M(x, y, t) * M(y, z, s) \leq M(x, z, t+s)$

(FM-5) $M(x, y, \cdot):(0, \infty) \rightarrow(0,1]$ is continuous.

Note that $M(x, y, t)$ can be thought of as the degree of nearness between $\mathrm{x}$ and $\mathrm{y}$ with respect to $\mathrm{t}$.

Definition 2.3. Two self maps A and B on a fuzzy metric space $(X, M, *)$ are said to be compatible if for all $t>$ $0, \lim \mathrm{n} \rightarrow \infty M\left(A B x_{n}, B A x_{n}, t\right)=1$ whenever $\left\{\mathrm{x}_{\mathrm{n}}\right\}$ is a sequence in $X$ such that $\lim _{\mathrm{n}} A x_{n}=\lim _{\mathrm{n}} B x_{n}=z$ for some $z \in X$.

Definition 2.4. Two self maps $f$ and $g$ on a fuzzy metric space $(X, M, *)$ are said to be weakly compatible if $f t=$ $g t$ for some $t \in X$ implies that $f g t=g f t$. It is well known fact that compatible maps are weak compatible but the converse is not true.

Definition 2.5. Two self maps $f$ and $g$ on a set $X$ are said to be owc if and only if there is a point $x \in X$ which is a coincidence point of $f$ and $g$ at which $f$ and $g$ commute. i.e., there exists a point $x \in X$ such that $f x=g x$ and $f g x$ $=g f x$. 
Definition 2.6. Two self maps $f$ and $g$ on a fuzzy metric space $(X, M, *)$ are said sub compatible if and only if there exists a sequence $\left\{\mathrm{x}_{\mathrm{n}}\right\}$ in $\mathrm{X}$ such that $\lim _{\mathrm{n}} f x_{n}=\lim _{\mathrm{n}} g x_{n}=z, z \in X$ and which satisfy $\lim _{\mathrm{n}} M\left(f g x_{n}, g f x n\right.$, $t)=1$ for all $t>0$.

Obviously two occasionally weakly compatible maps are sub compatible maps, however the converse is not true.

It is also interesting to see the following one way implication .

Commuting $\Rightarrow$ Weakly commuting $\Rightarrow$ Compatibility $\Rightarrow$ Weak

compatibility $\Rightarrow$ Occasionally weak compatibility $\Rightarrow$ Sub compatibility.

Definition 2.7. Two self maps $\mathrm{A}$ and $\mathrm{S}$ on a fuzzy metric space are called reciprocal continuous if $\lim n A S x_{n}=$ At and $\lim n S A x_{n}=S t$ for some $t \in X$ whenever $\left\{\mathrm{x}_{\mathrm{n}}\right\} n x$ is a sequence in $\mathrm{X}$ such that $\lim n A x_{n}=1 \mathrm{im}_{\mathrm{n}}$ $S x_{n}=t \in X$

Definition 2.8. Two self maps A and S on a fuzzy metric space are said to be sub sequentially continuous if and only if there exists a sequence $\left\{\mathrm{x}_{\mathrm{n}}\right\} \quad$ in $\mathrm{X}$ such that $\lim n A x_{n}=\lim n S x_{n}=t$ for some $t \in X$ and satisfy $\lim n$ $A S x_{n}=A t$ and $\lim n S A x_{n}=S t$.

Remark 1. If $\mathrm{A}$ and $\mathrm{S}$ are both continuous or reciprocally continuous then they are obviously sub sequentially continuous.

The next example shows that there exist sub sequentially continuous pairs of maps which are neither continuous nor reciprocally continuous.

\section{Results and Discussions}

Now, we prove the following result.

Theorem 1. Let $f, g, h$ and $k$ be four self maps on a fuzzy metric space $(X, M, *)$. If the pairs $(f, h)$ and $(g, k)$ are sub compatible and sub sequentially continuous, then (i) $f$ and $h$ have a coincidence point, (ii) $g$ and $k$ have a coincidence point.

Further, If

$\mathrm{M}(\mathrm{fx}, \mathrm{gy}, \mathrm{t}) \geq \phi\left[\min \left\{\begin{array}{l}M(h x, k y, t), M(f x, h x, t), M(g y, k y, t) \\ M(h x, g y, t), M(k y, f x, t), M(k x, h y, t)\end{array}\right\}\right]$

for all

$x, y \in X, \mathrm{t}>0$, where $\phi:[0,1] \rightarrow[0,1]$ is a continuous function such that $\phi(s)>s$ for

each $0<s<1$. Then $f, g, h$ and $k$ have a unique common fixed point in X.

Proof. Since the pairs $(f, h)$ and $(g, k)$ are sub compatible and sub sequentially continuous, therefore, there exist two sequences $\left\{\mathrm{x}_{\mathrm{n}}\right\}$ and $\left\{\mathrm{y}_{\mathrm{n}}\right\}$ in X such that $\lim _{\mathrm{n}} \mathrm{fx}_{\mathrm{n}}=\lim _{\mathrm{n}} \mathrm{hx}_{\mathrm{n}}=\lim _{\mathrm{n}} \mathrm{kx}_{\mathrm{n}}=u$ for some $u \in X$ and which satisfy

$\lim _{\mathrm{n}} M\left(f h x_{n,} h f x_{n}, t\right)=M(f u, h u, t)=1$,

$\lim _{\mathrm{n}} g y_{n}=\lim _{\mathrm{n}} k y_{n}=\lim _{\mathrm{n}} h y_{n}=\mathrm{v}$ for some $v \in X$ and which satisfy

$\lim _{\mathrm{n}} \mathrm{M}\left(\mathrm{gky}_{\mathrm{n}}, \mathrm{kgy}_{\mathrm{n}} \mathrm{t}\right)=M(g v k v, t)=1$.

Therefore, $f u=h u$ and $g v=k v$. i.e., $u$ is the coincidence point of $f$ and $h$ and $v$ is a

coincidence point of $g$ and $k$.

Now, using (1.1) for $\mathrm{x}=x_{n}$ and $y=\mathrm{y}_{\mathrm{n}}$, we get

$M\left(f x_{n}, g y_{n} t\right) \geq \phi\left(\min \left(\left(h x_{n}, k y_{n}, t\right), M\left(f x_{n}, h x_{n}, t\right), M\left(g y_{n}, k y_{n}, t\right), M\left(h x_{n}, g y_{n}, t\right), M\left(k y_{n}, f x_{n}, t\right), M\left(k x_{n}, h y_{n}, t\right)\right)\right.$

Letting $n \rightarrow \infty$

$M(u, v, t)=\phi(\min (M(u, v, t), M(u, u, t), M(v, v, t), M(u, v, t), M(v, u, t), M(u, v, t))$, a contradiction

hence $\mathrm{u}=\mathrm{y}$

Again using (1.1)

put $\mathrm{x}=\mathrm{u}$ and $\mathrm{y}=\mathrm{y}_{\mathrm{n}}$ we obtain

$\mathrm{M}\left(\mathrm{fu}, \mathrm{gy}_{\mathrm{n},} \mathrm{t}\right) \geq \phi\left(\min \left(M\left(h u, k y_{n}, t\right), M(f u, h u, t), M\left(g y_{n}, k y_{n}, t\right), M\left(h u, g y_{n}, t\right), M\left(k y_{n}, f u, t\right), M\left(k u, h y_{n}, t\right)\right)\right)$

Letting $n \rightarrow \infty$ 
$m(f u, v, t) \geq \phi(\min (M(f u, v, t), M(f u, h u, t), M(v, v, t), M(f u, v, t), M(f u, v, t), M(f u, v, t)))$

$m(f u, v, t) \geq \phi(\min (M(f u, v, t), 1,1, M(f u, v, t), M(f u, v, t), M(f u, v, t)))$

i.e

$M(f u, v, t)=\phi(M(f u, v, t))>M(f u, v, t)$ which yields $f u=v=u$ therefore

$u=v$ is a common fixed point of $f, g, h$ and $k$

for uniqueness let $w \neq u$ be another fixed point of $f, g, h, k$ then from (1.1) we have

$M(f u, g w, t) \geq \phi(\min (M(h u, k w, t), M(f u, h u, t), M(g w, k w, t), M(h u, g w, t), M(k w, f u, t), M(k u, h w, t))$

$=\phi(\min ((f u, g w, t), 1,1, M(f u, g w, t), M(g w, f u, t), M(f u, g w, t)))$

$=\phi(M(f u, g w, t)>M(f u, g w, t)$ which yields $w \neq$ uand hence prove the theorem.

Corollary 1. Let $f$ and $h$ be self maps on a fuzzy metric space $(X, M, *)$ such that the pairs $(f, h)$ is sub compatible and sub sequentially continuous, then (i) $f$ and $h$ have a coincidence point, Further, If

$\mathrm{M}(\mathrm{fx}, \mathrm{fy}, \mathrm{t}) \geq \phi(\min ((M(h x, h y, t) \cdot M(f x, h x, t), M(f y, h y, t), M(h x, f y, t), M(h y, f x, t)))$

for all

$x, y \in X, \mathrm{t}>0$, where $\phi:[0,1] \rightarrow[0,1]$ is a continuous function such that $\phi \quad(s)>s$ for each $0<s<1$. Then $f$ and $h$ have a unique common fixed point in $\mathrm{X}$.

Corollary 2. Let $f, g$ and $h$ be self maps on a fuzzy metric space $(X, M, *)$. Suppose

that the pairs $(f, h)$ and $(g, h)$ are sub compatible and sub sequentially continuous, then

(i) $f$ and $h$ have a coincidence point,

(ii) $g$ and $h$ have a coincidence point,

Further, If

$M($ hx hy $t) \geq \phi(\min (M(h x, h y, t), M(f x, h x, t) M($ gy hy $t) M($ hx gyt $), M(h y, f x, t))$

for all

$x, y \in X, \mathrm{t}>0$, where $\phi:[0,1] \rightarrow[0,1]$ is a continuous function such that $\phi(s)>s$ for each $0<s<1$. Then $f, g$ and $h$ have a unique common fixed point in $\mathrm{X}$.

Now, we furnish our theorem with example.

Example . Let $X=R$, equipped with usual metric $d$ and

$M(x y t)=t /(t+d(x, y))$

for all $x, y \in X, t>0$.

Define the maps $f, g, h$ and $k: X \rightarrow X$ as

$$
\begin{gathered}
f(x)=x, x \leq 1 \\
3 x+1, x>1 \\
h(x)=2 x-1, x \leq 1 \\
5 x-1, x>1 \\
g(x)=3-2 x, x \leq 1 \\
3, x>1 \\
k(x)=2, x<1 \\
3 x-2, x \geq 1
\end{gathered}
$$


Consider the sequences $\left\{X_{n}\right\}=\left\{Y_{n}\right\}=1-(1 / n)$

Then, clearly $f x_{n}, g x_{n}, h x_{n}$ and $k x_{n} \rightarrow 1$.

$f h\left(x_{n}\right)=f(1-(1 / n)=1-(1 / n) \rightarrow 1=f(1)$

and

$h f\left(x_{n}\right)=h(1-(1 / n)=1-(1 / n) \rightarrow 1=h(1)$

Thus $(f, h)$ is sub compatible and sub sequentially continuous.

Again,

$\operatorname{gk}\left(\mathrm{x}_{\mathrm{n}}\right)=\mathrm{g}(1-(1 / \mathrm{n}))=3-2(1-(1 / \mathrm{n})=(1+2 / \mathrm{n}) \rightarrow 1=\mathrm{g}(1)$

$\operatorname{kg}\left(. \mathrm{x}_{\mathrm{n}}\right)=\mathrm{g}(1+(1 / \mathrm{n}))=3(1+(1 / \mathrm{n}))=2(1+3 / \mathrm{n}) \rightarrow 1=\mathrm{k}(1)$

which shows that $(\mathrm{g}, \mathrm{k})$ is

compatible and sub sequentially continuous.

Also the condition (1.1) of our theorem is satisfied and ' 1 ' is unique common fixed point of $f, g, h$ and $k$.

\section{References}

[1] M. Abbas and B. E. Rhoades, Common fixed point theorems for hybrid pairs of occasionally weakly compatible mappings satisfying generalized condition of integral type, Fixed point theory appl., Art. ID 54101, 9pp.

[2] M. A. Al-Thagafi and N. Shahzad, A note on occasionally weakly compatible maps, Int. J. Math. Anal. 3(2)(2009), 55-58.

[3] H. Bouhadjera, A. Djoudi and B. Fisher, A unique common fixed point theorem for occasionally weakly compatible maps, Surveys in Mathematics and its Appli. 3(2008), 177-182.

[4] H. Bouhadjera and C. G. Thobie, Common fixed point theorems for pairs of sub compatible maps, Hal-00356516. 1(2009),1-16.

[5] H. Bouhadjera and C. G. Thobie, Common fixed point theorems for occasionally weakly compatible maps, ArXiv. 0812.373 [math. FA]. 2(2009): 123:131.

[6] H. Chandra and A. Bhatt, Fixed point theorem for occasionally weakly compatible maps in probabilistic semi-metric space, Int. J. Math. Anal. 3(12)(2008), 563-570.

[7] Z. Deng, Fuzzy pseudo-metric space, J. Math. Anal. Appl., 86(1982), 74-95.

[8] M. A. Erceg, Metric spaces in fuzzy set theory, J. Math. Anal. Appl. 69(1979), 205- 230

[9] G. Jungck and B. E. Rhoades, Fixed points for set valued functions without continuity, Indian J. Pure and Appl. Math., 29(3)(1998), 227-238.

[10] I. Kramosil and J. Michalek, Fuzzy metric and statistical metric spaces, Kybernetica, 11(1975), 326-334

[11] O. Kaleva and S. Seikkala, On fuzzy metric spaces, Fuzzy Sets Systems, 12(1984), 215-229.

[12] M. A. Khan and Sumitra, Common fixed point theorems for occasionally weakly compatible maps in fuzzy metric spaces, Far East Journal of Mathematical Sciences, 41(2) (2010), 285-293.

[13] R. P. Pant, Common fixed point theorems for contractive maps, J. Math. Anal. Appl., 226(1998), 251-258.

[14] S. Sessa, On a weak commutativity condition in fixed point considerations, Publ. Inst. Math. (Beograd) (N.S), 32(46)(1982),149153.

[15] L. A. Zadeh, Fuzzy Set. Information and Control. 8(1965), 338-353. 